\title{
Gleicht Schulbildung soziale Unterschiede aus?
}

\section{Leistungsunterschiede in der Kindheit nach sozioökonomischem Hintergrund}

\begin{abstract}
Bildungsungleichheit ist ein Hauptgrund dafür, dass Einkommensunterschiede über Generationen hinweg bestehen bleiben. Da das im Erwachsenenalter erzielte Einkommen mit kognitiven Fähigkeiten im Schulalter zusammenhängt, werden die schulischen Fähigkeiten von Kindern mit deren sozioökonomischem Hintergrund verglichen. Unsere Untersuchungen mit Paneldaten zu Schulkindern in Deutschland führen zu dem Ergebnis, dass Performanceunterschiede bereits zu Schulbeginn bestehen und die Lücken im Lauf der Schulzeit nicht geschlossen werden. Diese Ergebnisse sind relevant für politische Maßnahmen, die darauf zielen, die Einkommensmobilität zwischen Generationen zu erhöhen und Chancengleichheit zu schaffen. Frühkindliche Maßnahmen können helfen, bereits vor Schulbeginn bestehende Leistungsunterschiede zu verringern.
\end{abstract}

\begin{abstract}
Dem deutschen Grundgesetz zufolge gilt es, Chancengleichheit unabhängig von der sozialen Herkunft herzustellen (Art. 3 Absatz 3 GG). Insbesondere in Deutschland zeigen empirische Untersuchungen dennoch regelmäßig, dass Kinder mit niedrigerem sozioökonomischen Hintergrund schlechtere Testergebnisse als ihre Klassenkamerad*innen mit höherem sozialen Status erzielen. Im Kindesalter erworbene kognitive Fähigkeiten bilden die Grundlage für den späteren beruflichen Erfolg (Currie

(c) Der/die Autor(en) 2020. Open Access: Dieser Artikel wird unter der Creative Commons Namensnennung 4.0 International Lizenz (https:// creativecommons.org/licenses/by/4.0/deed.de) veröffentlicht.

Open Access wird durch die ZBW - Leibniz-Informationszentrum Wirtschaft gefördert.
\end{abstract}

Dr. Lukas Mergele ist wissenschaftlicher Mitarbeiter am ifo Zentrum für Bildungsökonomik des ifo Instituts München.

Johanna Raith ist Studentin an der Goethe-Universität Frankfurt im Master „International Economics and Economic Policy“.

Dr. Larissa Zierow ist am ifo Zentrum für Bildungsökonomik stellvertretende Leiterin. und Thomas, 1999). Der Einfluss des sozioökonomischen Hintergrunds auf kognitive Fähigkeiten impliziert daher, dass der soziale Status der Eltern auf die Kinder übertragen wird. Bereits in den 1960er Jahren wurden in den USA großflächig Daten erhoben, deren Untersuchung zeigt, dass der sozioökonomische Hintergrund in der frühkindlichen Entwicklung eine große Rolle spielt (Coleman, 1968). Besonders stark rezipiert sind die Ergebnisse von Cunha et al. (2006), die mit US-Daten eindrücklich nachweisen, dass Unterschiede in kognitiven Fähigkeiten nach sozioökonomischem Hintergrund bereits früh bestehen und sich über die Schullaufbahn hinweg nicht signifikant verändern. Diese Beobachtungen gehen mit Erkenntnissen aus der psychologischen Forschung zum Erwerb kognitiver Fähigkeiten einher. Diese zeigen, dass frühe kognitive und soziale Grundfähigkeiten die Basis für den späteren Erwerb höherer Fähigkeiten darstellen und später nicht ohne weiteres nachgeholt werden können. Die kritische Phase, in der diese Grundfähigkeiten vorwiegend geprägt werden, ist die frühe Kindheit (Knudsen et al., 2006).

Der Einfluss des sozioökonomischen Hintergrunds auf die Bildungschancen von Kindern fällt je nach Land sehr unterschiedlich aus. Die Ergebnisse der PISA-2015-Studie beispielsweise zeigen, dass der Anteil der Leistungsunterschiede, die durch den sozioökonomischen Status erklärt werden können, in Deutschland (ca. $15 \%$ ) um 5 Prozentpunkte höher liegt als in den USA (ca. 10\%) (BMF, 2019). Um näher zu ergründen, wie sich die Leistungsunterschiede nach sozioökonomischem Status in Deutschland im Verlauf der Schulkarriere entwickeln, untersuchen wir 
Kasten 1

Verwendete Daten

Wir verwenden das Nationale Bildungspanel (NEPS) Startkohorte 2, die Kindern vom Kindergarten (2011) bis zur 4. Klasse (2016) folgt, sowie die Startkohorte 3, die Kinder von der 5. (2011) bis zur 12. Klasse (2016) beobachtet. Diese Längsschnittstudie ist besonders geeignet, da sie erstmals in Deutschland dieselben Kinder über einen längeren Zeitraum begleitet und dabei Daten zu kognitiven Fähigkeiten und dem sozioökonomischen Hintergrund erhebt. Durch Zusammenfassen der Testungen in Mathematik und Naturwissenschaften zu einem MINT-Ergebnis beobachten wir in jeder Klassenstufe mathematisch-naturwissenschaftliche Fähigkeiten (außer in Klasse 8, wo keine solche Testung durchgeführt wurde). Wir betrachten Kinder, die von Klasse 1 bis 4 bzw. 5 bis 9 durchgängig an kognitiven Tests teilgenommen haben. Eine Limitation der Daten ist, dass insbesondere Kinder mit niedrigem sozioökonomischen Hintergrund häufiger nicht bis zum Studienende teilgenommen haben. Wir beschränken unsere Stichprobe auf durchgehend teilnehmende Kinder und reduzieren damit unsere Stichprobengröße von 7.958 auf 4.227 (Klasse 1 bis 4 ) bzw. von 5.094 auf 2.239 Kinder (Klasse 5 bis 9).

Als Indikatoren für den sozioökonomischen Hintergrund verwenden wir den Bildungsgrad der Eltern (ob zwei, eines oder keines der Elternteile einen Universitätsabschluss besitzt/besitzen) sowie eine dreistufige Gruppierung anhand der Zahl an Büchern, die eine Familie besitzt (weniger als 100 Bücher, 100 bis 200 Bücher, mehr als 200 Bücher). Die Bücherzahl als Indikator für den sozioökonomischen Hintergrund ist weitverbreitet, da das Maß stark mit dem Familieneinkommen korreliert und deswegen als Ersatz gilt, wenn Einkommensangaben wie in unserem Fall nur unvollständig vorhanden sind. In unserer Stichprobe mit verfügbaren Einkommensdaten liegt das durchschnittliche Einkommen von Elternhäusern mit den wenigsten Büchern bei 2.842 Euro netto pro Monat, das der mittleren Gruppe bei 3.540 Euro und das der höchsten Gruppe bei 4.593 Euro.

diese Zusammenhänge erstmals anhand einer deutschen Längsschnittstudie, die Kinder in der Grundschule und in der Sekundarstufe 1 nachverfolgt. In der Methodik und Darstellung folgen wir dabei dem Ansatz von Cunha et al. (2006) und verwenden Daten des Nationalen Bildungspanels (NEPS) (vgl. Kasten 1)'.

\section{Untersuchungsergebnisse zur Bildungsungleichheit}

Unsere Untersuchung zeigt, dass Bildungsungleichheit in Deutschland schon im frühen Grundschulalter besteht und im Laufe der Schuljahre bestehen bleibt. Sowohl in Sprachals auch MINT-Tests schneiden Kinder, die aus Familien mit geringerem sozioökonomischen Status stammen, schlechter ab als ihre Klassenkamerad*innen mit höherem sozioökonomischen Familienhintergrund. Abbildung 1a zeigt dies am Beispiel von Ergebnissen in MINT-Tests: Hier sind die durchschnittlich erreichten Perzentile je sozioökonomischer Gruppe über die Schullaufbahn abgebildet. Dabei zeigen die gestrichelten Linien die durchschnittlich erreichten Testperzentile von Kindern in den Klassenstufen 1 bis 4 und die durchgehenden Linien die Ergebnisse von Kindern in den Klassenstufen 5 bis 9 . Unterschiede sind bereits in der 1 .

1 Die Daten des NEPS wurden von 2008 bis 2013 als Teil des Rahmenprogramms zur Förderung der empirischen Bildungsforschung erhoben, das vom Bundesministerium für Bildung und Forschung (BMBF) finanziert wurde. Seit 2014 wird das NEPS vom Leibniz-Institut für Bildungsverläufe (LIfBi) an der Universität Bamberg in Kooperation mit einem deutschlandweiten Netzwerk weitergeführt.
Klasse zwischen allen drei Gruppen zu erkennen und bleiben bis zur 9. Klasse weitgehend konstant. Besonders groß ist die Lücke der oberen beiden Gruppen zur Gruppe mit dem niedrigsten sozioökonomischen Hintergrund. In Kompetenztests zur sprachlichen Entwicklung gibt es ähnliche Leistungsunterschiede, auch hier sind Performancelücken zwischen allen drei Gruppen und insbesondere zwischen den beiden unteren Gruppen zu erkennen.

Ähnliche Ergebnisse finden sich bei einer Gruppierung der Kinder nach dem Bildungsgrad ihrer Eltern (vgl. Abbildung 1b). Kinder, deren Eltern studiert haben, weisen höhere durchschnittliche Testergebnisse auf als Kinder mit weniger gebildeten Eltern. Dies ist in Abbildung $1 \mathrm{~b}$ anhand von MINT-Testergebnissen zu erkennen. Hier sind die durchschnittlich erreichten Performanceperzentile nach Akademikerstatus (keines, eines oder zwei der Elternteile mit Universitätsabschluss) dargestellt. Äquivalent zu Abbildung 1a sind auch hier bereits in der 1. Klasse Performancelücken zu erkennen. Die Größe dieser Lücken ändert sich bis zur neunten Klasse nur unwesentlich, und auch hier besteht der größte Unterschied zwischen den beiden oberen Gruppen und der unteren Gruppe. Ähnliche Ergebnisse werden bei Betrachtung der Sprachtestperformance deutlich.

\section{Mögliche Gründe für unterschiedliches Abschneiden}

Die bildungsökonomische Forschung legt nahe, dass die Gründe für diese Ergebnisse, soweit nicht biologisch de- 


\section{Abbildung 1}

\section{Testperformance in Mathe und Naturwissenschaften}

\section{in Perzentilen}

a. nach sozioökonomischem Hintergrund

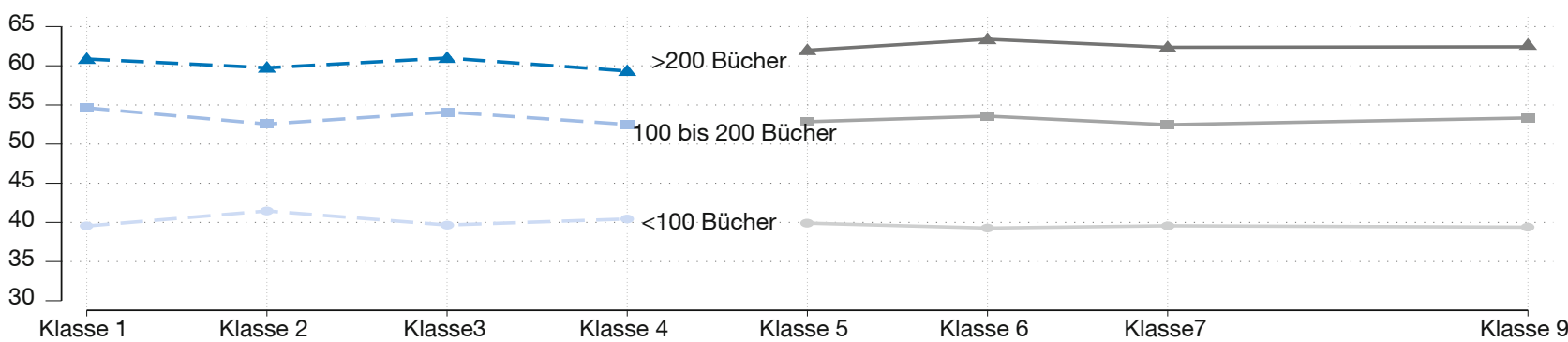

b. nach Akademikerstatus

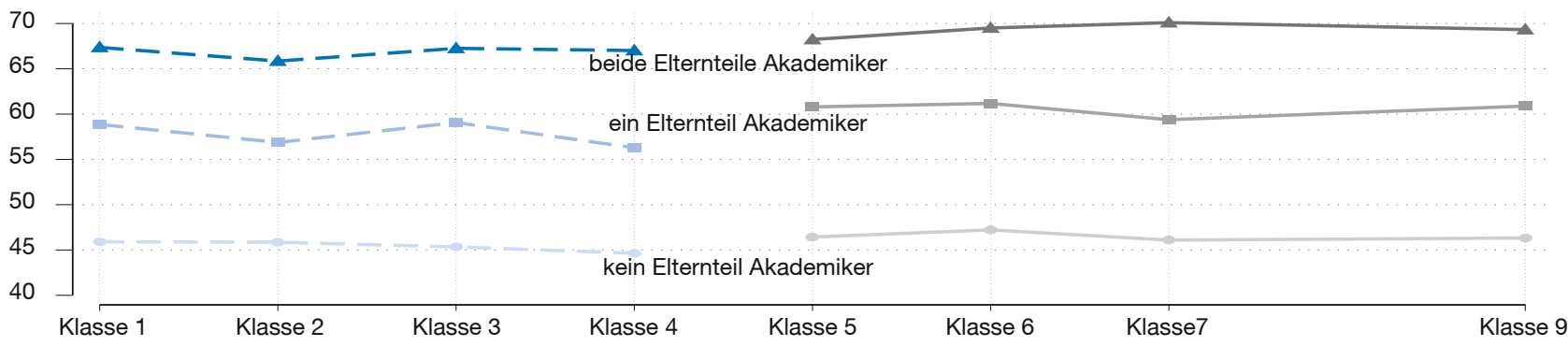

Anmerkungen: Kombination aus Startkohorte 2 (Klasse 1 bis 4 ) und Startkohorte 3 (Klasse 5 bis 12). Sample: Kinder, die von Klasse 1 bis 4 (5 bis 9 ) durchgängig an Tests teilgenommen haben. $\mathrm{N}=4.227$ (Klasse 1 bis 4); $\mathrm{N}=2.239$ (Klasse 5, 6, 7, und 9).

Quelle: NEPS Startkohorten 2 und 3 (2010 bis 2018), eigene Berechnungen.

terminiert, in unterschiedlichen äußeren Einflussfaktoren liegen können. Einige Bespiele aus der bestehenden Literatur sind Nachbarschaftsfaktoren, Familiengröße, Geburtsreihenfolge, das Schuleintrittsalter und verschiedene Eigenschaften der Eltern (Björklund und Salvanes, 2011). Anhand der Daten aus der NEPS Startkohorte 2 arbeiten wir einige Unterschiede zwischen Kindern aus unserer Stichprobe mit unterschiedlichem sozioökonomischen Hintergrund heraus. Zunächst ist ein deutlicher Unterschied im Besitz von Bildungsgütern festzustellen (vgl. Abbildung 2). Kinder mit höherem sozioökonomischen Hintergrund haben im Schnitt gut vier der abgefragten Kategorien an Bildungsgütern wie Wörterbücher, Bibliotheksausweis, Kunstwerke, Gedichte und klassische Literatur im häuslichen Umfeld zur Verfügung, während Familien mit geringem sozioökonomischen Hintergrund nur knapp drei dieser Kategorien besitzen.

Auch hinsichtlich der in Abbildung 3 dargestellten Ausgaben der Familien für außerschulische Aktivitäten ihrer Kinder ist ein deutlicher Unterschied von mehr als 20 Euro pro Woche zwischen der obersten und untersten sozioökonomischen Gruppe zu erkennen. Wenn der Preis das Spektrum der Aktivitäten bzw. deren Qualitäten (z. B. hinsichtlich Betreuungsverhältnis) wiedergibt, könnte hierin eine der Ursachen für die Lücke in kognitiven Fähigkeiten liegen. Ein weiterer relevanter Faktor für die Entwicklung von Fä- higkeiten ist die Zeit, die Kinder mit ihren Eltern verbringen, sowie die Qualität dieser Zeit. Kinder mit geringerem sozioökonomischen Hintergrund verbringen durchschnittlich mehr Zeit mit ihren Eltern. Dieser Unterschied beträgt insgesamt eine Stunde und 24 Minuten pro Woche und $42 \mathrm{Mi}-$ nuten mit Blick auf schulische Unterstützung. Die Zeit, die Kinder wöchentlich bei außerschulischen Aktivitäten mit ihren Eltern verbringen, ist zwischen allen Gruppen identisch (vgl. Abbildung 4). In der Qualität der Zeit ist kaum ein Unterschied zwischen den Gruppen festzustellen: Eltern

\section{Abbildung 2}

Menge an Wissensgütern' im Heimbesitz $\mathrm{N}=4.210$

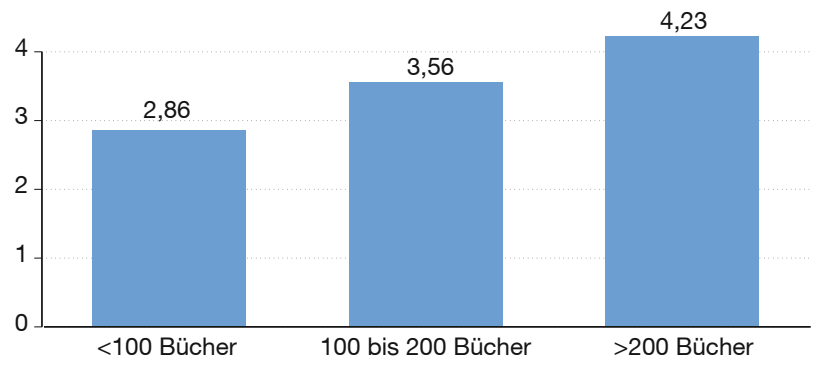

1 Kunstwerke, Bibliotheksausweis, Gedichte, Wörterbuch, klassische Literatur.

Quelle: NEPS Startkohorte 2. 
Abbildung 3

Ausgaben für außerschulische Aktivitäten

$\mathrm{N}=3.374$

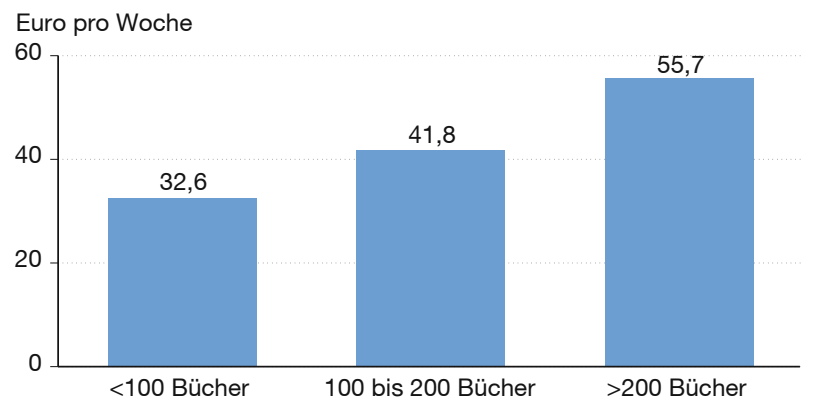

Quelle: NEPS Startkohorte 2.

aller Gruppen beschäftigen sich im Schnitt ein- bis mehrmals pro Woche mit ihren Kindern mit bildenden Aktivitäten wie z.B. Vorlesen. Allenfalls eine leichte Qualitätszunahme ist mit höherem sozioökonomischen Status verbunden. Ein größerer Unterschied liegt in der Zeit, die Kinder mit Fernsehen oder Computer- und Konsolenspielen verbringen. Abbildung 5 zeigt, dass Kinder der niedrigsten sozioökonomischen Gruppe im Schnitt rund eine Stunde mehr pro Woche mit diesen Aktivitäten verbringen. Zusätzlich kann sich die Einstellung der Eltern zu schulischem Erfolg auf Kinder übertragen. Allerdings stellen wir keinen Unterschied in der Wichtigkeit fest, die Eltern schulischer Leistung und schulischen Abschlüssen beimessen. Insofern können die aufgezeigten Leistungsunterschiede durch unterschiedliche elterliche Einstellungen zu Bildung nicht erklärt werden.

\section{Mögliche Maßnahmen gegen Bildungsungleichheit}

Unsere Ergebnisse stellen heraus, dass Leistungsunterschiede zwischen Kindern in Deutschland bereits bei Schuleintritt bestehen. Um Bildungsungleichheit zu senken, können politische Maßnahmen entweder a) in der frühen Kindheit ansetzen, sodass die frühen Leistungsunterschiede gar nicht erst aufkommen, oder b) das Schulsystem reformieren, sodass es diese Diskrepanzen im Nachhinein ausgleicht. Dabei zeigt Heckman (2000), dass frühe Interventionen effizienter sind als Maßnahmen, die auf junge Erwachsene abzielen. Die von ihm analysierten Interventionen weisen Nutzen-Kosten-Verhältnisse von bis zu 9:1 auf. Dabei sind die Programme, die besonders früh einsetzen, besonders effektiv. Im Folgenden beschreiben wir einige politische Maßnahmen, welche die Leistungsunterschiede im jungen Alter beeinflussen können.

- Sozial schwache Familien können ihre Kinder nicht in demselben Maß unterstützen und fördern wie Eltern mit höherem Familieneinkommen. Der Effekt von finanzieller Unterstützung benachteiligter Familien auf
Abbildung 4

Wie viel Zeit Eltern mit ihren Kindern verbringen

$\mathrm{N}=3.552$ (allgemein); 3.837 (schulische Unterstützung); 3.413 (außerschulische Aktivitäten)

Stunden pro Woche

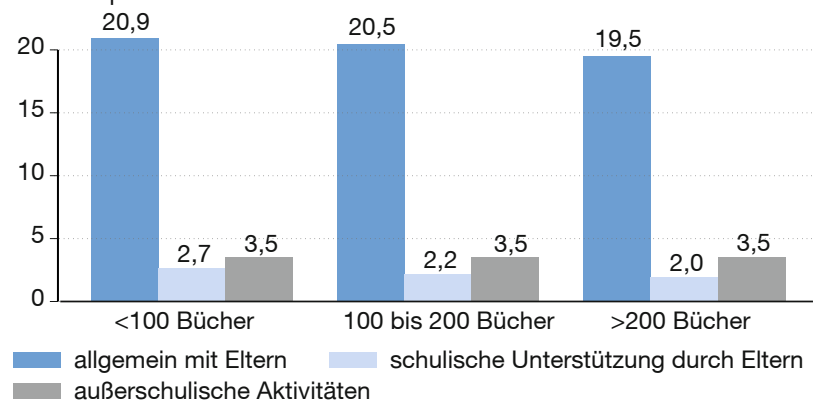

Quelle: NEPS Startkohorte 2.

die kognitiven Fähigkeiten von deren Kindern zeigt, inwieweit geringere kognitive Performance auf monetäre Engpässe und die daraus resultierende geringere Förderung zurückzuführen ist. Dahl und Lochner (2012) zeigen für die USA, dass eine Einkommenserhöhung um 1.000 US-\$ mit um $6 \%$ höheren Testergebnissen in Mathematik und Lesen verbunden ist. Dabei ist der Effekt für Kinder mit gering gebildeten Müttern größer. Der Effekt auf kognitive Leistungen durch Steigerungen des Familieneinkommens ist umso höher, je früher sie im Leben des Kindes stattfinden (Duncan et al., 2011).

- Eine mögliche Maßnahme, die häusliche Lernumwelt benachteiligter Kinder zu verbessern, ist Elterntraining. Dies zielt darauf ab, Eltern zu informieren, welche Methoden besonders geeignet sind, um Kinder im Erwerb kognitiver Fähigkeiten zu fördern. Avvisati et al. (2014) zeigen, dass Aufklärung von Eltern über optimale schulische Unterstützung in der sechsten Klasse dabei hilft, Schulnoten und Motivation der Schüler*innen zu verbessern. Auch die von Cunha et al. (2006) untersuchten Interventionsprogramme beinhalten ähnliche Komponenten. So werden im Abecedarian Project wie auch im Perry Preschool Project Hausbesuche durchgeführt, welche die Bereitstellung zusätzlicher häuslicher Bildungsaktivitäten unterstützen und die Eltern animieren, sich stärker an der Bildung ihrer Kinder zu beteiligen.

- Der Besuch eines Kindergartens mit hoher Betreuungsqualität hat das Potenzial, Bildungsungleichheit zu verringern (z.B. Cornelissen et al., 2018). Untersuchungen zeigen, dass eine Steigerung der Kindergartenqualität - in Form von mehr kognitiver Förderung verbunden mit dem Eingehen auf individuelle Lernbedürfnisse - die Bildungsresultate langfristig positiv beeinflusst (Lehrl et al., 2017). Insbesondere Förderung im Bereich Sprache hat einen positiven Einfluss auf kognitive Fähigkeiten (Joo et al., 2020). 


\section{Abbildung 5}

Wie viel Zeit Kinder mit Fernsehen, Computer- oder Konsolenspielen verbringen

\section{$\mathrm{N}=333$}

Stunden pro Woche

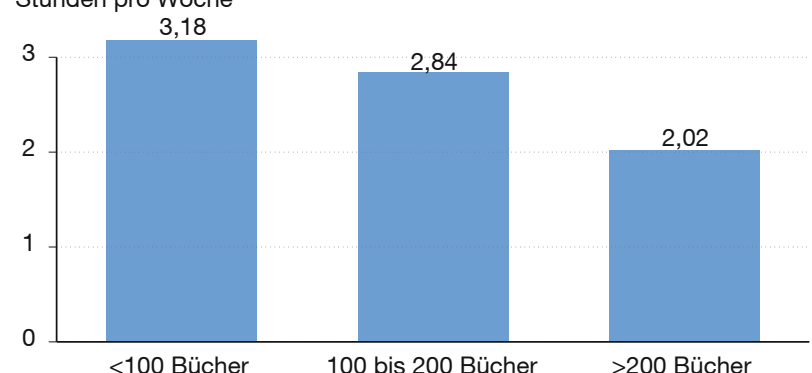

Quelle: NEPS Startkohorte 2.

- Ein weiterer Unterschied zwischen Kindern unterschiedlicher sozialer Hintergründe liegt in Sozialkompetenzen, die im Erwerb kognitiver Fähigkeiten eine wichtige Rolle spielen. Geringere Sozialkompetenzen von Kindern werden in der Forschung häufig mit schwächerem Sozialverhalten der Eltern und in einer geringeren Zahl sozialinteraktiver Tätigkeiten begründet. Mentoring-Programme, in denen benachteiligte Kinder verstärkt fördernden Eins-zu-eins-Interaktionen mit Mentor*innen ausgesetzt werden, können dazu beitragen, Unterschiede im Sozialverhalten zwischen sozioökonomischen Gruppen auszugleichen (Kosse et al., 2020).

\section{Fazit}

Bildungsgerechtigkeit spielt eine wichtige Rolle für die soziale Mobilität in einer Gesellschaft. Die Ergebnisse zeigen, dass in Deutschland Performancelücken zwischen Kindern unterschiedlichen sozioökonomischen Hintergrunds bereits früh vorhanden sind und diese sich über die Schullaufbahn hinweg nicht wesentlich verändern. Das bedeutet zum einen, dass bereits vor Schulbeginn wichtige Faktoren den Werdegang eines Kindes beeinflussen. Zum anderen implizieren die Ergebnisse auch, dass das deutsche Schulsystem die bestehende Performancelücke zwar nicht verringert, gleichzeitig aber ein weiteres Auseinanderdriften der kognitiven Fähigkeiten unterschiedlicher sozioökonomischer Gruppen verhindert. Für geeignete Reformen bedeutet dies: Gezielte Maßnahmen in der frühen Kindheit - Mentoring-Programme, Elterntraining, qualitativ hochwertige Förderung im Kindergarten - könnten zu einer Verringerung der Leistungslücken zum Schulbeginn führen. Da Performancelücken sich über die Schullaufbahn nur wenig verändern, könnte dies eine langfristige Steigerung sozialer Mobilität bewirken.

\section{Literatur}

Avvisati, F., M. Gurgand, N. Guyon und E. Maurin (2014), Getting parents involved: A field experiment in deprived schools, Review of Economic Studies, 81(1), 57-83.

Björklund, A. und K. G. Salvanes (2011), Education and family background: Mechanisms and policies, Handbook of the Economics of Education, Bd. 3, Elsevier, 201-247.

BMF (Bundesministerium der Finanzen) (2019), Soziale Ungleichheit und inklusives Wachstum im internationalen Vergleich, Monatsbericht des Bundesfinanzministeriums, Mai.

Cornelissen, T., C. Dustmann, A. Raute und U. Schönberg (2018), Who benefits from universal child care? Estimating marginal returns to early child care attendance, Journal of Political Economy, 126(6), 23562409 .

Coleman, J. S. (1968), Equality of educational opportunity, Integrated Education, 6(5), 19-28.

Cunha, F., J. J. Heckman, L. Lochner und V. D. Masterov (2006), Interpreting the evidence on life cycle skill formation, Handbook of the Economics of Education, 1, 697-812.

Currie, J. und D. Thomas (1999), Early test scores, socioeconomic status and future outcomes, National Bureau of Economic Research Working Paper, Nr. 6943.

Dahl, G. B. und L. Lochner (2012), The impact of family income on child achievement: Evidence from the earned income tax credit, American Economic Review, 102(5), 1927-1956.

Duncan, G. J., P. A. Morris und C. Rodrigues (2011), Does money really matter? Estimating impacts of family income on young children's achievement with data from random-assignment experiments, Developmental psychology, 47(5), 1263

Heckman, J. J. (2000), Policies to foster human capital, Research in Economics, 54(1), 3-56.

Joo, Y. S., K. Magnuson, G. J. Duncan, H. S. Schindler, H. Yoshikawa und K. M. Ziol-Guest (2020), What works in early childhood education programs? A meta-analysis of preschool enhancement programs, Early Education and Development, 31(1), 1-26.

Knudsen, E. I., J. J. Heckman, J. L. Cameron und J. P. Shonkoff (2006), Economic, neurobiological, and behavioral perspectives on building America's future workforce, Proceedings of the National Academy of Sciences, 103(27), 10155-10162.

Kosse, F., T. Deckers, P. Pinger, H. Schildberg-Hörisch und A. Falk (2020), The formation of prosociality: causal evidence on the role of social environment, Journal of Political Economy, 128(2), 434-467.

Lehrl, S., K. Kluczniok, H.-G. Rossbach und Y. Anders (2017), Long Term Persistence of Preschool Intervention on Children's Mathematical Development: Results from the German Model Project „Kindergarten of the Future in Bavaria“, Global Education Review, 4(3), 70-87.

Title: Does School Education Balance out Social Differences?

Abstract: Educational inequality is a major reason why income disparities persist across generations. With our analysis of panel data on school children in Germany, we show that performance differences by socioeconomic background are already present before children start formal schooling. These gaps then persist throughout children's school careers. We find differences in important family characteristics by socioeconomic background, which might contribute to the early and persistent gap in cognitive abilities. These results are relevant for policy measures aimed at increasing intergenerational income mobility and creating equal opportunities. Combining our results with prior research suggests that policy interventions should target early childhood to minimise performance gaps at school entry age.

JEL Classification: 124, I28, J13 